\title{
Mid-infrared spectral variability for compositionally similar asteroids: Implications for asteroid particle size distributions
}

\author{
P. Vernazza ${ }^{\mathrm{a}, *}$, B. Carry ${ }^{\text {b,c }}$, J. Emery ${ }^{\mathrm{d}}$, J.L. Hora ${ }^{\mathrm{e}}$, D. Cruikshank ${ }^{\mathrm{f}}$, R.P. Binzel ${ }^{\mathrm{g}}$, J. Jackson ${ }^{\mathrm{h}}$, J. Helbert ${ }^{\mathrm{i}}$, \\ A. Maturilli ${ }^{\mathrm{i}}$ \\ ${ }^{a}$ Research and Scientific Support Department, European Space Agency, Keplerlaan 1, 2201 AZ Noordwijk, The Netherlands \\ ${ }^{\mathrm{b}}$ Laboratoire d'Etudes Spatiales et d'Instrumentation en Astrophysique, Observatoire de Paris, 5 Place Jules Janssen, Meudon F-92195, France \\ ' European Southern Observatory, Alonso de Cordova 3107, Vitacura, Casilla 19001, Santiago de Chile, Chile \\ ${ }^{\mathrm{d}}$ Department of Earth and Planetary Sciences, Knoxville, TN 37996-1410, USA \\ ${ }^{\mathrm{e}}$ Harvard-Smithsonian Center for Astrophysics, Cambridge, MA 02138, USA \\ ${ }^{\mathrm{f}}$ NASA Ames Research Center, Mail Stop 245-6, Moffett Field, CA 94035, USA \\ ${ }^{\mathrm{g}}$ Department of Earth, Atmospheric and Planetary Sciences, MIT, Cambridge, MA 02139, USA \\ ${ }^{\mathrm{h}}$ Institute for Astrophysical Research, Boston University, Boston, MA 02215, USA \\ ${ }^{\mathrm{i}}$ Institute for Planetary Research, DLR, Rutherfordstrasse 2, 12489 Berlin, Germany
}

\section{A R T I C L E I N F O}

\section{Article history:}

Received 20 July 2009

Revised 22 December 2009

Accepted 9 January 2010

Available online 21 January 2010

\section{Keywords:}

Asteroids, Surfaces

Spectroscopy

Mineralogy

Meteorites

\begin{abstract}
A B S T R A C T
We report an unexpected variability among mid-infrared spectra (IRTF and Spitzer data) of eight S-type asteroids for which all other remote sensing interpretations (e.g. VNIR spectroscopy, albedo) yield similar compositions. Compositional fitting making use of their mid-IR spectra only yields surprising alternative conclusions: (1) these objects are not "compositionally similar" as the inferred abundances of their main surface minerals (olivine and pyroxene) differ from one another by $35 \%$ and (2) carbonaceous chondrite and ordinary chondrite meteorites provide an equally good match to each asteroid spectrum.

Following the laboratory work of Ramsey and Christensen (Ramsey, M.S., Christensen, P.R. [1998]. J. Geophys. Res. 103, 577-596), we interpret this variability to be physically caused by differences in surface particle size and/or the effect of space weathering processes. Our results suggest that the observed asteroids must be covered with very fine $(<5 \mu \mathrm{m})$ dust that masks some major and most minor spectral features. We speculate that the compositional analysis may be improved with a spectral library containing a wide variety of well characterized spectra (e.g., olivine, orthopyroxene, feldspar, iron, etc.) obtained from very fine powders. In addition to the grain size effect, space weathering processes may contribute as well to the reduction of the spectral contrast. This can be directly tested via new laboratory irradiation experiments.
\end{abstract}

(c) 2010 Elsevier Inc. All rights reserved.

\section{Introduction}

Spectroscopy in the visible near-infrared range (VNIR, 0.4$2.5 \mu \mathrm{m}$ ) has proven to be a powerful tool for constraining the surface composition of certain asteroid classes (e.g. A-, S- and V- classes; see DeMeo et al. (2009) for a definition of taxonomic classes over the VNIR range). Importantly, it has allowed researchers to determine the linkages between specific meteorite classes and their parent bodies. Several VNIR links have been proposed that are helping scientists understand the formation and evolution of the Solar System bodies:

(1) McCord et al. (1970) showed a spectroscopic match between the basaltic HED meteorites and (4) Vesta.

\footnotetext{
* Corresponding author. ESA-ESTEC (RSSD SCI), Keplerlaan 1, 2201 AZ Noordwijk, The Netherlands. Fax: +31 (0)71565 4697.
}

E-mail address: pierre.vernazza@esa.int (P. Vernazza).
(2) Cruikshank and Hartmann (1984) were the first to identify plausible parent bodies for olivine-rich meteorites such brachinites or pallasites. More recently, Sunshine et al. (2007) proposed additional parent bodies for these same meteorites.

(3) Vernazza et al. (2008) showed that the Flora family (semimajor axis $a=2.2 \mathrm{AU}$ ) is the most likely source region for $L L$ chondrites ( $8 \%$ of all recovered meteorites).

(4) Nesvorný et al. (2009) showed that the shocked $L$ chondrite meteorites, which represent about two-thirds of all $L$ chondrite falls and $25 \%$ of all falls, may be fragments of the Gefion family (semimajor axis $a=2.8 \mathrm{AU}$ ).

(5) Vernazza et al. (2009) showed that (21) Lutetia and (97) Klotho are plausible parent bodies for enstatite chondrite meteorites while (201) Penelope, (250) Bettina and (337) Devosa are compatible with the properties of mesosiderites. 
With one exception (enstatite chondrites), all proposed links were established mainly because of the presence of diagnostic VNIR spectral bands (due to the presence of silicates such as olivine and pyroxene) in both meteorite and asteroid spectra. While some clear progress has been reached in linking olivine- and/or pyroxene-rich meteorites to their parent asteroids, little progress has been achieved for other compositions (e.g. for the B and C taxonomic classes) whose VNIR spectra are mostly featureless. As an example, the surface composition of two of the largest asteroids ( 1 Ceres and 2 Pallas) remains elusive.

A solution to this problem may be reached by extending the wavelength coverage. The $2.9-4.0 \mu \mathrm{m}$ range is sensitive to the presence of water/water ice as well as the presence of carbonates (Rivkin et al., 2002, 2006) but these components cannot explain the overall composition of a given asteroid when present $(<10 \%$ of the composition). Thus, the extended wavelength range accessible from the ground that may help us constraining the surface composition of asteroids is the mid-IR range $(8-13 \mu \mathrm{m})$.

Mid-infrared spectroscopy has been quite successful in constraining the surface composition of Mars (e.g. Milam et al., 2004, 2007). For example, Milam et al. (2004) constrained the abundance of feldspar (and its composition) on the surface of Mars by deconvolution modeling of mid-IR spectra. Such result could not have been obtained from the VNIR range where the detection of feldspar is difficult. These martian results clearly revealed the power of mid-IR spectroscopy for detecting the presence of some minerals that are difficult to detect at shorter wavelengths. In principle, one may expect the same success with asteroids.

In this paper, we investigate methods for obtaining mineralogical information (for asteroids) in this wavelength range. For the target selection, we decided to focus our attention on S-type asteroids because we can consistently determine the relative abundance $( \pm \sim 5 \%)$ of their main surface components (olivine, orthopyroxene) within the VNIR range as demonstrated for both laboratory meteorites and asteroids (Vernazza et al., 2008; Binzel et al., 2009). Constraining in advance the relative abundance of their main surface components should simplify the interpretation of their emissivity spectra. Specifically, we compare the mid-IR spectral properties of S-type asteroids which have, within 5\%, nearly identical relative abundances of olivine and orthopyroxene based on compositional analysis in the VNIR range. By observing the properties of "compositionally identical" asteroids, as interpreted within the VNIR range, we evaluate the results of "controlled" measurements extended into the mid-IR.

We first present the observing protocol and the data reduction process, followed by result and discussion subsections.

\section{Observations and data reduction}

We present here observations obtained from the ground-based NASA Infrared Telescope Facility (IRTF), as well as observations collected within the science archive of the Spitzer Space Telescope.

\subsection{Near-infrared}

NIR measurements for seven S-type asteroids were acquired remotely from MIT (Cambridge, USA) on the 3-m NASA IRTF located on Mauna Kea, Hawaii. The instrument SpeX (Rayner et al., 2003) was utilized in prism mode to obtain measurements covering the wavelength range $0.8-2.5 \mu \mathrm{m}$ in one exposure. The observing and reduction procedures are well described by Rivkin et al. (2004).

\subsection{Mid-infrared}

Mid-infrared spectral measurement from 8 to $14 \mu \mathrm{m}$ for three S-type asteroids ((7) Iris, (11) Parthenope and (43) Adriane, see Table 1) were obtained remotely from the Paris Observatory on the IRTF (Bus et al., 2002) on March 16 and July 30, 2008. The instrument MIRSI (Deutsch et al., 2003) was used in the 8-14 $\mu \mathrm{m}$ range with the $1.2^{\prime \prime}$ slit which was aligned in the North-South direction.

Because the sky and the telescope are bright sources at midinfrared wavelengths (due to their thermal emission), it is mandatory to subtract their contributions to the recorded signal. We thus followed the standard protocol for mid-infrared observations ("chop \& nod", see Lim et al. (2005a,b) for an extensive description of the procedure). We acquired spectra for the target and the nearby sky background by chopping the secondary mirror at a few Hertz (typically $4 \mathrm{~Hz}$ ). However, since the light path is not the same for the two chopping positions, some sky residuals were still present after chopping. This is why we placed the target at several positions on the detector by nodding the entire telescope. The combination of these nodded frames ultimately allowed us minimizing the contribution from the sky and the telescope.

Standard techniques for infrared spectroscopy reduction were used in order to obtain the final spectra. These included: (i) the extraction of the spectra from the frames by summing at each wavelength the flux inside a region centered on the spectrum and delimited by a 3- $\sigma$ dispersion of the Gaussian profile of the spectrum (perpendicularly to the dispersion direction) and (ii) the wavelength calibration. Finally, all acquired spectra for a given object (asteroid, star) were combined by a median combining procedure.

The last step included: (1) the isolation of the asteroid spectrum, (2) the removal of the telluric absorptions, (3) the stellar spectral shape and (4) the asteroid thermal emission. First, in order to isolate the asteroid's spectrum, we needed to estimate the overall response function by measuring the spectrum of a mid-infrared calibration star under similar atmospheric conditions. For each asteroid spectrum, we observed a standard star close to the asteroid in time and airmass. We chose among bright stars whose infrared spectra were well known so that we could correct later the spectral artefacts introduced by the star (e.g., Cohen et al., 1992, 1999; Cohen and Davies, 1995).

Since the division of the asteroid spectra by the standard star spectra did not complete telluric corrections, we used an atmospheric modeling program to improve the quality of the correction.

Ground-based thermal infrared observations are heavily affected by variability in atmospheric water vapor, ozone and other gases as well as background emission. Therefore, good telluric correction is critical to accurate reduction of thermal IR spectra. In many cases, appropriate telluric standards are either too faint for good correction, or too widely separated for a good air mass match.

Table 1

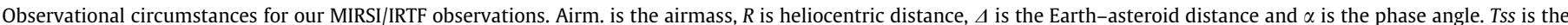
temperature of the asteroid surface at the subsolar point that was derived from our STM model.

\begin{tabular}{|c|c|c|c|c|c|c|c|c|c|}
\hline Object & $\begin{array}{l}\text { Observation } \\
\text { date (UT) }\end{array}$ & $\begin{array}{l}\text { Start time-end } \\
\text { time (UT) }\end{array}$ & Airm. & $\begin{array}{l}\text { Integration time } \\
\text { per segment (s) }\end{array}$ & Standard star & $R(\mathrm{AU})$ & $\Delta(\mathrm{AU})$ & $\alpha\left(^{\circ}\right)$ & Tss (K) \\
\hline 7 Iris & 2008-03-19 & $13: 10-14: 36$ & $1.29-1.70$ & $30-70$ & Alpha Boo, Vega & 2.83 & 1.90 & 9.3 & 248.4 \\
\hline 11 Parthenope & 2008-07-30 & $11: 34-13: 40$ & $1.26-1.65$ & $40-50$ & Beta Peg & 2.21 & 1.20 & 4.0 & 285.5 \\
\hline 43 Adriane & $2008-07-30$ & $14: 51-15: 21$ & $1.15-1.23$ & 50 & Beta Peg & 1.93 & 1.07 & 22.0 & 301.7 \\
\hline
\end{tabular}




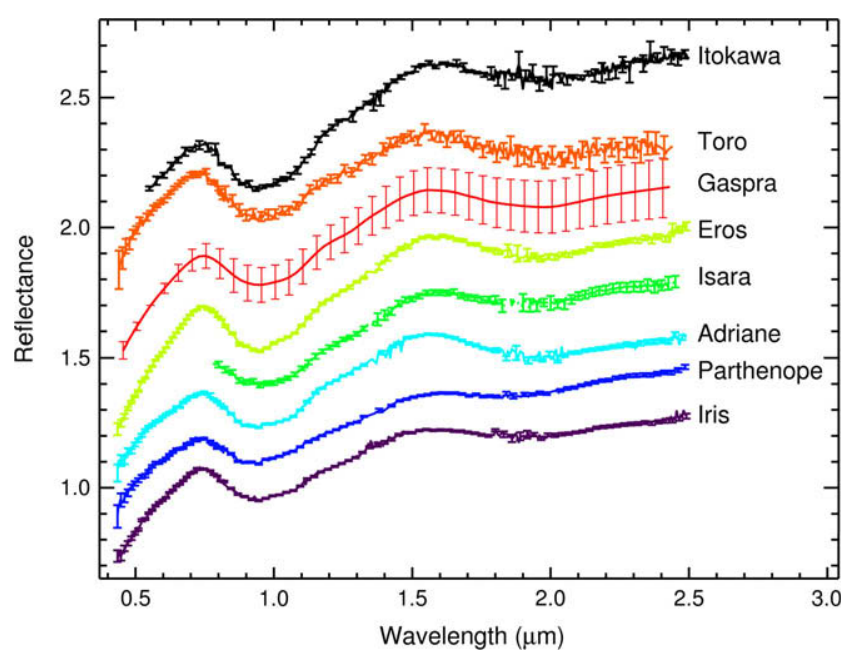

Fig. 1. VNIR reflectance spectra of 7 Iris, 11 Parthenope, 43 Adriane, 433 Eros, 951 Gaspra (since we do not have Gaspra's NIR spectrum we use the mean spectrum of several Flora family member spectra), 1685 Toro and 25,143 Itokawa as well as the NIR spectrum of 364 Isara. The NIR portion of the spectra was acquired with the IRTF; the visible portion of the spectrum was available from SMASS (see Bus and Binzel, 2002a,b). All these objects belong to the S-type class following the Bus and/ or the new Bus-DeMeo taxonomy (Bus, 1999; Bus and Binzel, 2002a,b; Demeo et al., 2008)

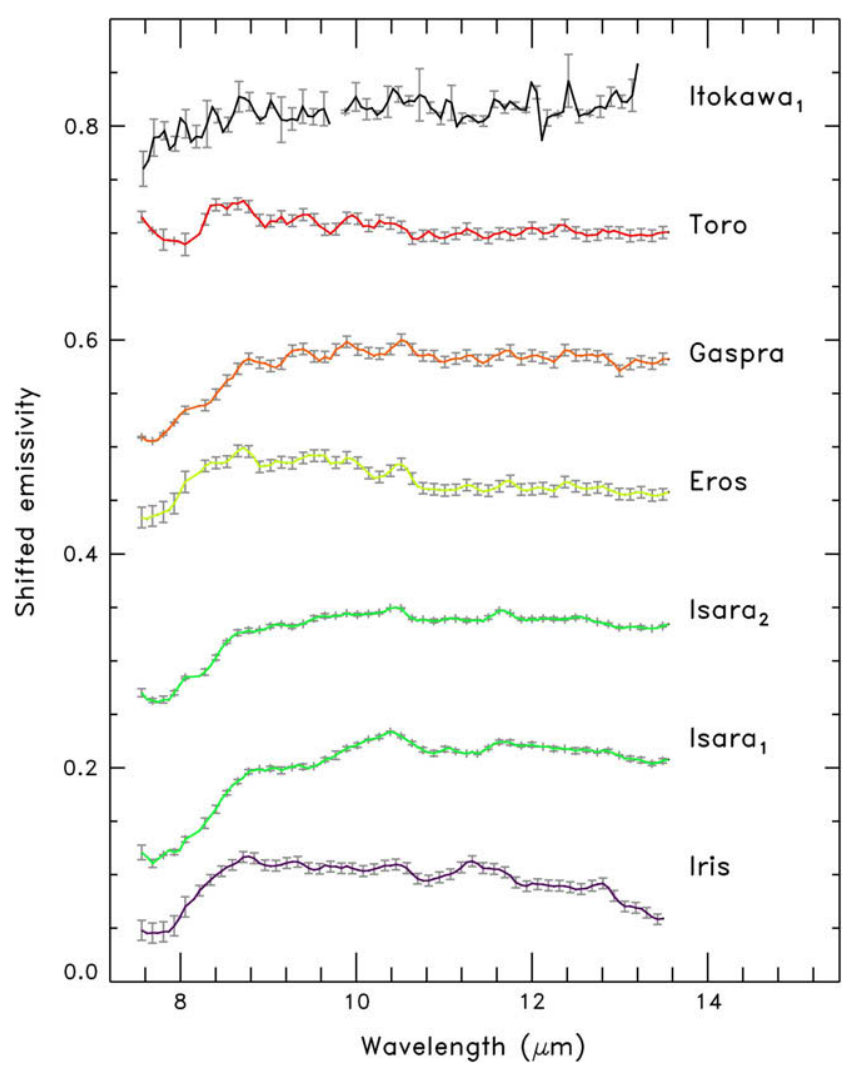

Fig. 2. Spitzer emissivity spectra of 7 Iris, 364 Isara, 433 Eros, 951 Gaspra, 1685 Toro and 25,143 Itokawa created by dividing the measured SED by the bestfit STM for each object. Isara was observed twice and we therefore show both spectra. Itokawa has been observed six times and we just show the data for the first observation. The spectra for the other observing dates are very similar and even noisier.

In addition, the infrared backgrounds can change during observations of target objects, making accurate telluric correction using observed standards difficult. Therefore, in addition to the observa-

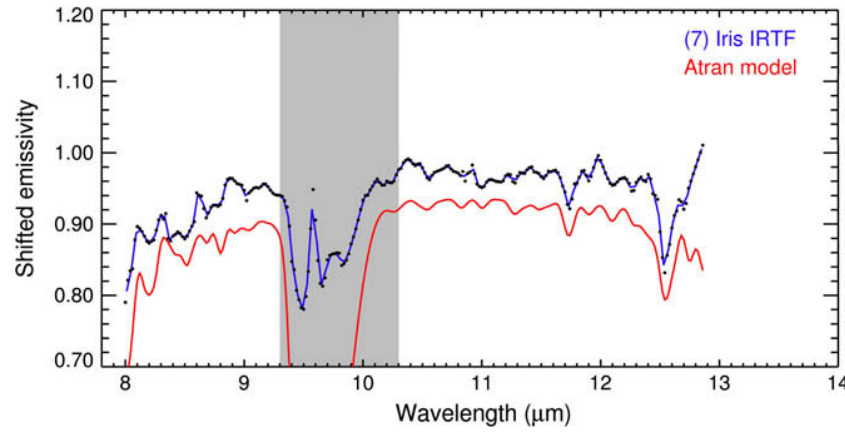

Fig. 3. Comparison of the IRTF (Mirsi) emissivity spectrum of (7) Iris before the telluric correction with an Earth's atmospheric transmission spectrum computed with ATRAN. The displayed (7) Iris spectrum has been corrected for both the thermal emission and the shape of the observed star. We do not display the error bars accompanying the shape correction here (they appear in the following figure) to highlight the high signal to noise ratio of our observations. The comparison shows that the numerous features seen in the (7) Iris spectrum are also seen in the Earth's transmission spectrum. This highlights that a simple division by a standard star observed close in time and airmass to the asteroid observation is simply not enough to remove the telluric features.

tion of telluric standards, ATRAN, an atmospheric modeling program developed by Lord (1992), can be used to generate artificial telluric calibrator spectra at the target's zenith angle and air mass. ATRAN models allow tweaking of water vapor overpressure and other atmospheric conditions to produce optimized telluric corrections for each target.

We simulated with the ATRAN model the Earth atmospheric transmission spectrum for each observation at each different air mass and corrected both the star and the asteroid spectrum by multiplying their spectrum by the transmission spectrum (the transmission spectra were binned to the resolving power of Mirsi). In Fig. 3, we show the spectrum of (7) Iris before the ATRAN correction together with a typical transmission spectrum of the Earth atmosphere. The multiple features that can be seen in Iris' spectrum before the ATRAN correction are retrieved in the Earth transmission spectrum.

After completing the telluric corrections, the quotient was corrected for the stellar spectral slope and features. As noticed by Cohen et al. (1992), a SiO absorption band is present in the stellar spectra. Thus while the division of the asteroid spectrum by the standard star spectrum removes the telluric absorptions, it also introduces stellar features. Finally, in order to produce the final emissivity for each object we removed the thermal emission from each object spectrum. Several thermal emission models exist, from the simple STM (Lebofsky et al., 1986) to the refined TPM (Mueller and Lagerros, 1998; Lagerros, 1998). In the present work, we modeled the thermal emission using the STM model. (The STM fitting method is well described in Emery et al. (2006a,b).) The final emissivity spectrum was created by dividing the SED by the modeled thermal continuum.

Lastly, given the uncertainty of the correction of both the telluric absorptions and the stellar spectral shape, we retrieved for comparison public Spitzer spectra of several S-type asteroids taken with IRS (InfraRed Spectrograph, Houck et al., 2004) using the Leopard software (see Table 2). We selected the so-called Basic Calibrated Data (BCD) which is a 2-D output. After background removal from the BCD images, we extracted the 1-D spectra from these images. (See Section 3 by Emery et al. (2006a,b) for a detailed description of the method.) Finally, we removed the thermal emission of each asteroid in order to produce its emissivity (see Table 3 for the STM best-fit parameters for both the IRTF and Spitzer observations). Figs. 2 and 4 show the Spitzer and IRTF spectra after the thermal correction. 
Table 2

Observational circumstances for the Spitzer observations. $R$ is heliocentric distance, $\Delta$ is the Earth-asteroid distance and $\alpha$ is the phase angle.

\begin{tabular}{llllll}
\hline Object & $\begin{array}{l}\text { Observation } \\
\text { date (UT) }\end{array}$ & $\begin{array}{l}\text { Start time-end } \\
\text { time (UT) }\end{array}$ & $R(\mathrm{AU})$ & $\Delta(\mathrm{AU})$ & $\alpha\left(^{\circ}\right)$ \\
\hline 7 Iris & $2005-03-22$ & $15: 40-15: 52$ & 2.91 & 2.72 & 20.2 \\
364 Isara & $2005-04-15$ & $08: 49-09: 07$ & 2.07 & 1.90 & 29.0 \\
364 Isara (1) & $2005-04-18$ & $07: 51-08: 09$ & 2.07 & 1.93 & 28.8 \\
433 Eros & $2004-09-30$ & $00: 52-01: 01$ & 1.31 & 0.96 & 50.6 \\
951 Gaspra & $2006-02-03$ & $21: 40-21: 50$ & 1.97 & 1.27 & 26.3 \\
1685 Toro & $2004-07-14$ & $14: 39-14: 45$ & 1.14 & 0.38 & 61.6 \\
25,143 Itokawa & $2007-05-03$ & $23: 07-23: 14$ & 1.00 & 0.52 & 75.3 \\
\hline
\end{tabular}

\section{Results}

\subsection{VNIR}

\subsubsection{Compositional analysis of S-type asteroids in the VNIR range} Asteroids belonging to the S-complex ( $\mathrm{Q}, \mathrm{S}, \mathrm{Sa}, \mathrm{Sq}, \mathrm{Sr}$, Sv; see Bus (1999) and Bus and Binzel (2002a,b) for the taxonomic classification in the visible) share similar spectral properties in the VNIR range based on a simple visual inspection (e.g. Gaffey et al., 1993; DeMeo et al., 2008, and Fig. 1 of this paper). Their spectra are characterized by the presence of (1) a moderate to steep reddish slope shortward of $0.7 \mu \mathrm{m},(2)$ absorption features around 1 and $2 \mu \mathrm{m}$ due to the presence of both olivine and orthopyroxene, (3) a neutral to steep slope of the continuum across the $1 \mu \mathrm{m}$ band. These same characteristics are also shared by the $\mathrm{V}, \mathrm{R}$ and $\mathrm{O}$ classes. What makes the S-complex distinct is its spectral similarity (without considering the slope difference induced by aging effects) with the spectra of the most common meteorites, namely the ordinary chondrites (OCs, $80 \%$ of the recovered falls).

In this wavelength range, the relative abundance of the two main minerals (about $70 \%$ of the whole composition (Hutchison, 2004)) namely olivine (ol) and orthopyroxene (opx) can be inferred by using (1) the Cloutis et al. (1986) method or (2) a scattering model (Hapke, 1993 or Shkuratov et al., 1999) combined with the space weathering model developed by Brunetto et al. (2006). Method 1 has been widely used (e.g. Gaffey et al., 1993, see review by Gaffey et al. (2002)). Method 2 combines both the Shkuratov or Hapke models and the Brunetto model. It allows constraining the surface composition of a given asteroid (to be compared with those of ordinary chondrites) and its space weathering degree (see Brunetto et al. (2006), Vernazza et al. (2008) and Binzel et al. (2009) for direct applications off this technique). Using the model on well characterized OCs, Vernazza et al. (2008) and Binzel et al. (2009) determined its precision to be $\pm 5 \%$.

\subsubsection{Compositional analysis of our S-type sample}

In Fig. 1 we show the VNIR spectra of seven S-type asteroids ((7) Iris, (11) Parthenope, (43) Adriane, (433) Eros, (951) Gaspra, (1685)

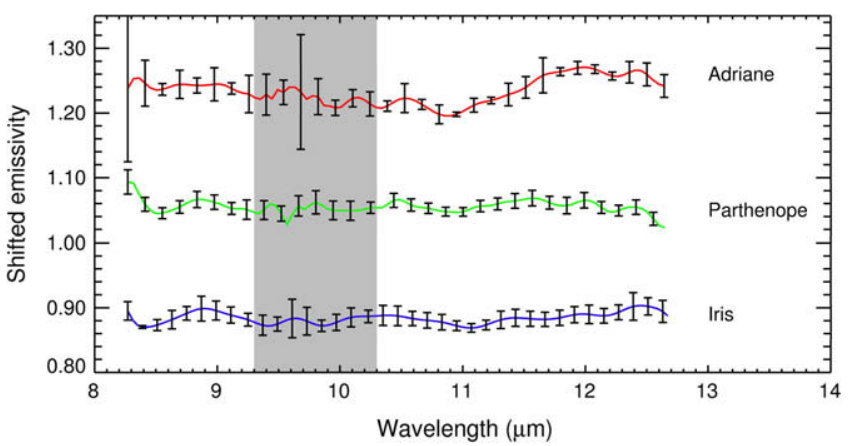

Fig. 4. IRTF (Mirsi) emissivity spectra of (7) Iris, (11) Parthenope and (43) Adriane created (1) by dividing the measured SED by the bestfit STM for each object, (2) after correction of the star spectral shape, and (3) after an additional telluric correction with the ATRAN model. The error bars $(3-\sigma)$ include both the errors originating from the correction of the star spectral shape and from the ATRAN telluric correction. Spectra were shifted vertically for clarity.

Toro and $(25,143)$ Itokawa) as well as the NIR spectrum of (364) Isara. Using method 2 mentioned above, we calculated the relative abundance of olivine and orthopyroxene [ol/ol +opx] for each asteroid that we report in Table 4. It appears that all the objects have a very similar $[\mathrm{ol} / \mathrm{ol}+\mathrm{opx}]$ value (in the $75-80 \%$ range) that closely resembles the [ol/ol $+\mathrm{opx}$ ] value of $L L$ chondrite meteorites ( $75 \pm 3 \%$, see Vernazza et al., 2008). (For (364) Isara we derived the [ol/ol + opx] value based on the NIR spectrum only. The NIR range is sufficient for deriving the [ol/ol + opx] value, the accuracy dropping from $\pm 5 \%$ to $\pm 7 \%$.) The next step consisted in using mid-IR spectral data for these same objects and determining the [ol/ ol + opx] value from this range.

\subsection{Mid-IR}

\subsubsection{Mid-IR laboratory measurements}

Laboratory studies of the mid-infrared spectral properties of powdered meteorites and minerals have revealed that their spectra display a wide variety of features that can be used for their identification (e.g., Lyon, 1964; Hunt and Logan, 1972; Salisbury et al., 1991; Christensen et al., 2000; Maturilli et al., 2006, 2008; Helbert et al., 2007). These include reststrahlen features, which occur as reflectance peaks, the Christiansen feature, which occurs as a trough in reflectance and the transparency feature which results in a broad reflectance maximum or emittance minimum (Salisbury et al., 1991). Importantly, laboratory studies have shown that the grain size of the measured sample greatly affects the spectral shape of the recorded spectrum (e.g., Hunt and Vincent, 1968; Hunt and Logan, 1972; Moersch and Christensen, 1995; Mustard and Hays, 1997). As an example, the overall spectral contrast gets significantly reduced with decreasing particle sizes (Mustard and Hays,

Table 3

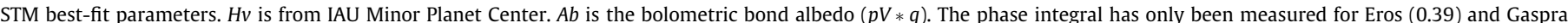

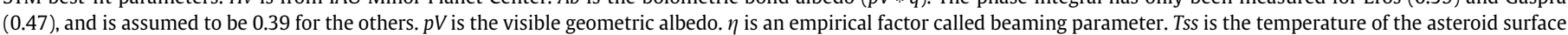
at the subsolar point that was derived from our STM model.

\begin{tabular}{|c|c|c|c|c|c|c|}
\hline Object & $H v$ & Radius (km) & $A b$ & $p V$ & $\eta$ & Tss (K) \\
\hline 7 Iris (Spitzer) & 5.53 & 119.5 & 0.075 & 0.193 & 1.03 & 231.3 \\
\hline 7 Iris (IRTF) & 5.53 & 113.1 & 0.083 & 0.212 & 0.806 & 248.4 \\
\hline 11 Parthenope (IRTF) & 6.55 & 78.7 & 0.067 & 0.171 & 0.77 & 285.5 \\
\hline 43 Adriane (IRTF) & 7.93 & 34.7 & 0.097 & 0.247 & 0.784 & 301.7 \\
\hline 364 Isara (Spitzer) & 9.88 & 14.77 & 0.090 & 0.230 & 1.05 & 271.8 \\
\hline 364 Isara (1) (Spitzer) & 9.88 & 14.51 & 0.093 & 0.239 & 0.93 & 279.7 \\
\hline 433 Eros (Spitzer) & 11.18 & 7.44 & 0.107 & 0.274 & 0.79 & 364.5 \\
\hline 951 Gaspra (Spitzer) & 11.65 & 6.93 & 0.096 & 0.205 & 0.94 & 285.7 \\
\hline 1685 Toro (Spitzer) & 14.32 & 2.00 & 0.082 & 0.210 & 2.08 & 308.7 \\
\hline 25,143 Itokawa (Spitzer) & 19.2 & 0.190 & 0.101 & 0.256 & 1.75 & 342.6 \\
\hline
\end{tabular}


Table 4

Comparison between the compositions of our S-type sample derived from the VNIR and mid-IR range.

\begin{tabular}{|c|c|c|c|c|c|}
\hline \multirow[t]{2}{*}{ Asteroid } & \multicolumn{2}{|c|}{ Data reference $^{a}$} & \multirow[t]{2}{*}[ol/(ol+opx)]{$\%$ VNIR } & \multirow[t]{2}{*}[\mathrm{ol}/(\mathrm{ol}+\mathrm{opx})]{$\%$ Mid-IR (case b) } & \multirow[t]{2}{*}[ol/(ol+opx)]{$\%$ Mid-IR (case c) } \\
\hline & $\mathrm{V}$ & NIR & & & \\
\hline 7 & $\mathrm{~S}$ & 20 February 2004 & 76.5 & $48^{\mathrm{b}} \mid 46^{\mathrm{c}}$ & $66^{\mathrm{b}} \mid 100^{\mathrm{c}}$ \\
\hline 11 & $\mathrm{~S}$ & 13 November 2005 & 79.5 & $36^{\mathrm{c}}$ & $43^{\mathrm{c}}$ \\
\hline 43 & $\mathrm{~S}$ & 22 November 2005 & 76 & $65^{c}$ & $87^{c}$ \\
\hline 364 & & 03 October 2007 & 77 & $61^{\mathrm{b}} \mid 47^{\mathrm{b}}(1)$ & $55^{\mathrm{b}} \mid 41^{\mathrm{b}}(1)$ \\
\hline 433 & $\mathrm{~S}$ & 17 August 2002 & 79 & $37^{\mathrm{b}}$ & $58^{\mathrm{b}}$ \\
\hline 951 & $\mathrm{~S}$ & & 79 & $49^{b}$ & $41^{\mathrm{b}}$ \\
\hline 1685 & $\mathrm{~S}$ & 09 March 2005 & 77.5 & $26^{\mathrm{b}}$ & $37^{\mathrm{b}}$ \\
\hline 25,143 & $\mathrm{~S}$ & 28 March 2001 & 75 & $38^{\mathrm{b}}$ & $43^{b}$ \\
\hline
\end{tabular}

S: smass.

a For observations reported here, we give the observation date (UT). All near-infrared (NIR) data were obtained using the NASA IRTF at Mauna Kea, Hawaii.

b With Spitzer.

c With IRTF.

1997) while the position of the emission features changes. Since fine grained particles $(<50 \mu \mathrm{m})$ dominate particle size distributions of asteroid surfaces (Dollfus et al., 1977), one may expect weak features in the asteroid emissivity spectra. This little spectral contrast expected for asteroid surfaces further requires that, in the asteroidal case, data with high SNR (>100) should be recorded for meaningful interpretation (Lim et al., 2005a,b).

Another important field of investigation that has been conducted via laboratory studies concerns the implementation of a spectral deconvolution model for mid-IR data. Deconvolution provides a relatively straightforward and computationally quick method of assessing the mineral assemblages of a multimineralic surface (Ramsey and Christensen, 1998). It has been demonstrated through compositional analysis that thermal infrared spectra represent linear combinations of the abundance of the individual surface components (Lyon, 1964; Gillespie, 1992; Thomson and Salisbury, 1993; Ramsey and Christensen, 1998; Ramsey et al., 1999; Ramsey and Fink, 1999; Feely and Christensen, 1999; Hamilton and Christensen, 2000). Therefore, if spectra of the pure endmembers are known, mixture spectra (e.g. asteroid emissivity spectra) can then be deconvolved through a least squares linear fit resulting in a percentage of each input end-member plus a measure of the model quality (rms). However, the success of this technique appears to be strongly dependent on the particle size of the observed surface.

Ramsey and Christensen (1998) applied a linear retrieval algorithm to (a) 70 mineral mixtures having particle diameters of 250-500 $\mu \mathrm{m}$, and (b) 21 mineral mixtures with the particle diameter varying form $<10 \mu \mathrm{m}$ to $500 \mu \mathrm{m}$ to test the limits of the model at decreasing particle sizes. Note that the only sample components prepared at less than $250 \mu \mathrm{m}$ were quartz and andesine. In case (a) they found that the assumption of linear mixing is valid and enables mineral percentage prediction within $5 \%$ on average. In case (b) they found that spectral mixing remains essentially linear down to grain sizes of $\sim 60 \mu \mathrm{m}$. Below this threshold, they found that the linear retrieval algorithm fails using a single size fraction for the end-member. However, with spectra of the appropriate size fraction, the linearity continues through the $10-20 \mu \mathrm{m}$ size fractions. For particle sizes below $10 \mu \mathrm{m}$ where non-linear volume scattering dominates, the retrieval algorithm fails.

\subsubsection{Mid-IR asteroidal measurements}

Broad-band observations around 10 and $20 \mu \mathrm{m}$, together with visual measurements, have been used to determine the radii and bond albedo of a number of asteroids (e.g. Allen, 1970; Morrison, 1973, 1974, 1977). At the same epoch, observations with higher spectral resolution have also been performed (e.g. Gillett and Merrill, 1975; Hansen, 1976). These investigators found no deviations from gray body emission greater than their observational errors (10\% level), i.e. they found no evidence of emission features in their spectra. Using a high-sensitivity spectrometer in the $8-13 \mu \mathrm{m}$ range $(\Delta \lambda / \lambda=0.017-0.027)$, Feierberg et al. (1983) observed six main-belt asteroids and found that two (19) Fortuna and (21) Lutetia, appeared to show silicate emission features. Their findings suggested that the $8-13 \mu \mathrm{m}$ spectral region may be a potential source of compositional information for asteroids. However, spectrophotometric observations by Green et al. (1985) in the $8-13 \mu \mathrm{m}$ range did not confirm Feierberg's et al. (1983) findings.

Successful results were achieved by the space telescope IRAS whose data analysis provided the albedo and radii of 2228 mainbelt asteroids via broad-band photometry at $12 \mu \mathrm{m}, 25 \mu \mathrm{m}$, $60 \mu \mathrm{m}$ and $100 \mu \mathrm{m}$ (Matson et al., 1986; Dermott et al., 1988; Tedesco et al., 1988, 2002).

Several years later, Dotto et al. $(2000,2002)$ and Barucci et al. (2002) reported spectroscopic observations (5.8-11.6 $\mu \mathrm{m}$ range) for large main-belt asteroids obtained by the ESA space telescope ISO. They compared the obtained spectra with laboratory spectra of various minerals and meteorites in order to constrain the asteroid compositions but did not find good spectral matches.

Recently, Lim et al. (2005a,b) reported the $8-13 \mu \mathrm{m}$ spectral properties of 29 asteroids from a wide variety of visible to nearIR spectral classes (MIDAS survey). They found evidence of emissivity features with spectral contrast greater than $5 \%$ for a single asteroid, namely (1) Ceres. In their best data (for (4) Vesta), they achieved sufficiently high signal to noise ratios to detect subtle features $(2-3 \%)$. The insufficient SNR for the rest of their data prevented them from a mineralogical characterization of their sample. Their main conclusion is that future observers may be able to find mineralogical features on these and other main-belt asteroids provided that their spectral uncertainties can be brought below $2 \%$.

Emery et al. (2006a,b) presented the mid-IR Spitzer spectra of three Trojans. Comparisons with meteorites, mineral emissivity and inverted reflectance libraries, linear mixtures of these minerals, and Hapke-Mie modeling point to fine-grained silicates to best explain their mid-IR data. However, Emery et al. (2006a,b) state that they are not able at this point to determine the specific silicate mineralogy implied by the Trojan emissivity spectra.

Overall, mid-IR spectroscopic observations of asteroids and larger 'atmosphere less bodies' such as the Moon and Mercury have been the subject of numerous campaigns over the recent years showing the growing interest of scientists for the output that can be obtained from this wavelength range (Lim et al., 2005a, 2006, 2008, 2009; Emery et al., 2006a, 2008; Lim and Emery, 2006; Donaldson Hanna et al., 2007; Kozlowski et al., 2007; Barucci et al., 2008; Donaldson Hanna and Sprague, 2009; Marchis et al., 2008; Sprague et al., 2009). 
3.2.3. Compositional analysis of our S-type sample

3.2.3.1. Spectral heterogeneity of the data. Fig. 2 shows the Spitzer emissivity spectra for six S-type asteroids (three main-belt asteroids and three near-Earth asteroids) and Fig. 4 shows the IRTF (MIRSI) emissivity spectra for three large S-type main-belt asteroids. For the Spitzer data, the best SNR was achieved for 364 Isara whereas for the IRTF data, (7) Iris and (11) Parthenope show an equally high SNR.

We first performed a visual inspection of the spectra and searched for characteristic features. We also compared the spectra to each other. We perform the data description for each telescope (Spitzer and IRTF) separately.

Overall, the Spitzer spectra look quite similar even though small differences can be noticed. The Spitzer spectra show all evidence of a moderate reddish slope shortward of $8.7 \mu \mathrm{m}$ besides $(25,143)$ Itokawa and perhaps (1685) Toro. Beyond $\sim 8.7 \mu \mathrm{m}$ which appears to be the Christiansen feature for all spectra, we observe two kinds of spectra. The first group - that comprises (7) Iris, (364) Isara, (433) Eros and maybe (951) Gaspra - shows evidence of a flat continuum on which small emission features located around $\sim 10.5 \mu \mathrm{m}$ and $\sim 11.7 \mu \mathrm{m}$ are superimposed. Note that the $11.7 \mu \mathrm{m}$ feature does not appear clearly from the (433) Eros and (951) Gaspra data. A lower SNR for these objects may be the cause. The second group includes $(1685)$ Toro and $(25,143)$ Itokawa. Their spectra are completely flat over the entire range (note that $(25,143)$ Itokawa's noisy data may hide small features).

As far as the IRTF data is concerned, two objects appear to have a similar spectrum ((7) Iris and (11) Parthenope). Both objects display a rather flat spectrum over the entire $8-13 \mu \mathrm{m}$ range which looks very similar to the $(25,143)$ Itokawa and (1685) Toro spectra. (43) Adriane's spectrum looks slightly different and displays a broad bump (like a $V$ that has been reversed) from 11 to $13 \mu \mathrm{m}$. This basic spectral comparison (for the Spitzer and IRTF data) shows that in the mid-IR range, moderate spectral differences for objects having a very similar composition can be expected.

Finally, the only object observed by both MIRSI and Spitzer is Iris. In Fig. 5, we show a direct comparison of those spectra. Overall the spectra agree pretty nicely over the entire $8-13 \mu \mathrm{m}$ range. This comparison is very promising for future ground-based observations now that Spitzer has run out of cryogen and therefore cannot measure any more MIR spectra.

3.2.3.2. Compositional fitting with a linear retrieval algorithm. Our next and final step consisted in fitting the composition of all objects using the mid-IR spectral data. From the VNIR range we reached very clear conclusions namely that (1) all objects have nearly identical [ol/ol + opx] values (in the $0.75-0.8$ range) and (2) we could link these compositionally "identical" objects to a specific meteorite class namely the $L L$ chondrite class. Thus, the first objective of our mid-IR compositional analysis was to test whether we would be able to converge towards similar consistent conclusions in this much wider wavelength range $(8-13 \mu \mathrm{m}$ versus $0.4-2.4 \mu \mathrm{m})$.

To answer this question we applied a linear retrieval algorithm (see Sprague et al. (2009) for an exhaustive description of the method) to all S-type spectra (Spitzer \& IRTF). The only free parameters of the algorithm were the abundance of each mineral. We did not include a term that effectively allows the spectral contrast to vary. We used as end-members (a) all available meteorite reflectance spectra from the ASTER spectral library, (b) spectra for the two main minerals found in ordinary chondrite meteorites, namely olivine and low-Ca pyroxene reflectance spectra from ASTER as well as olivine and low-Ca pyroxene emissivity spectra from the BED library (Helbert et al., 2007; Maturilli et al., 2006, 2008), and (c) spectra for all mineralogical constituents

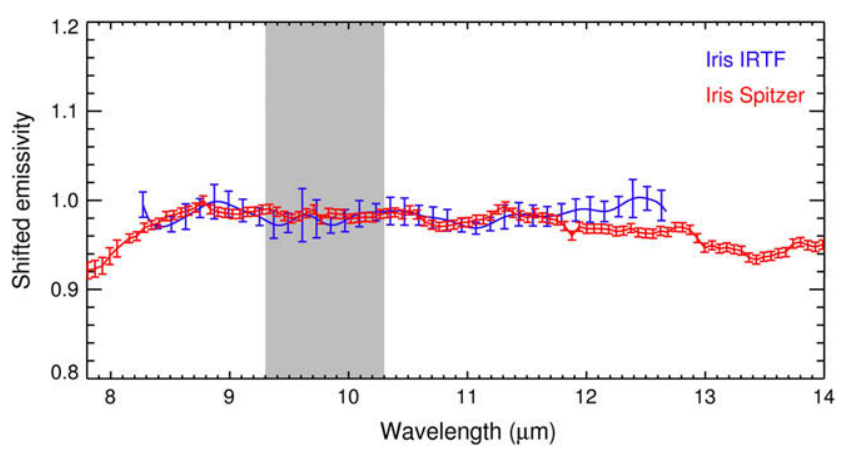

Fig. 5. Comparison between both the MIRSI (IRTF) and Spitzer emissivity spectra for 7 Iris.

found in ordinary chondrite meteorites, namely olivine, low-Ca pyroxene and feldspar reflectance spectra from ASTER as well as olivine, low-Ca pyroxene and feldspar emissivity spectra from the BED library, iron spectra from the RELAB database and chromite spectra from ASTER. We give a list of the end-members in the figure captions. For the meteorite spectra, we ran the code for the following subclasses: ordinary chondrites, carbonaceous chondrites, HEDs and achondrites. The biconical reflectance spectra from the ASTER and RELAB libraries were converted to emissivity by using Kirchoff's law: $1-5 r=\varepsilon$ (see Lim et al., 2005a,b). Finally, all emissivity spectra were normalized to unity at a given wavelength $(11 \mu \mathrm{m})$.

Figs. 6 and 7 show the fitting results for the (a), (b), and (c) cases listing the relative abundance of (a) $H, L$ and $L L$ meteorites for OCs, the relative abundance of $\mathrm{CM}, \mathrm{CI}$ and $\mathrm{CV} / \mathrm{CO}$ meteorites for $\mathrm{CCs}$, (b) the relative abundance of olivine and low-Ca pyroxene and (c) the relative abundance of olivine, low-Ca pyroxene, feldspar, iron and chromite. It does not list the fitting results for the HEDs and achondrites subclasses because in these cases the derived rms was too high (i.e. bad fits). In all three cases (a, b, and c), the fitting yields divergent interpretations for the compositions. Focusing on the relative abundance of olivine and low-Ca pyroxene (cases b and c), a $35 \%$ and $30 \%$ (b and c respectively) difference in composition is observed among the objects which have been observed with Spitzer whereas a $29 \%$ and 19\% (b and c respectively) difference is observed among the objects which have been observed with IRTF. In case c, we also note a huge variation of the abundance of other components depending on the target asteroid. For example, the fraction of feldspar is around $4 \%$ for (951) Gaspra whereas it reaches 33\% for (7) Iris. Furthermore, an association between these S-types and $L L$ chondrites appears excluded from these results. It even appears that CCs provide in some cases a better match to the asteroid spectrum than OCs. A first conclusion is that with a linear retrieval algorithm, consistent compositional analysis is problematic for this wavelength range even with high SNR data (e.g. (364) Isara with Spitzer or (7) Iris with IRTF). Here, inferred abundances of olivine and low-Ca pyroxene show on average a $\sim 30 \%$ difference (using both the IRTF and Spitzer values) with the abundances derived from the VNIR range and a $\sim 32 \%$ difference using the Spitzer values only. This supports the conclusions of Ramsey and Christensen (1998) for small grained surfaces derived from laboratory studies.

A last interesting case concerns the fitting results for both Spitzer observations of (364) Isara. This object offers the opportunity to compare the variability of the results of the linear deconvolution for a given object. As listed in Table 4, we find a $14 \%$ difference in the [ol/ol +opx] value for both datasets. This value allows us to define a confidence level of $\sim 20 \%$ (we take a rather large upper limit) for our fitting results. 


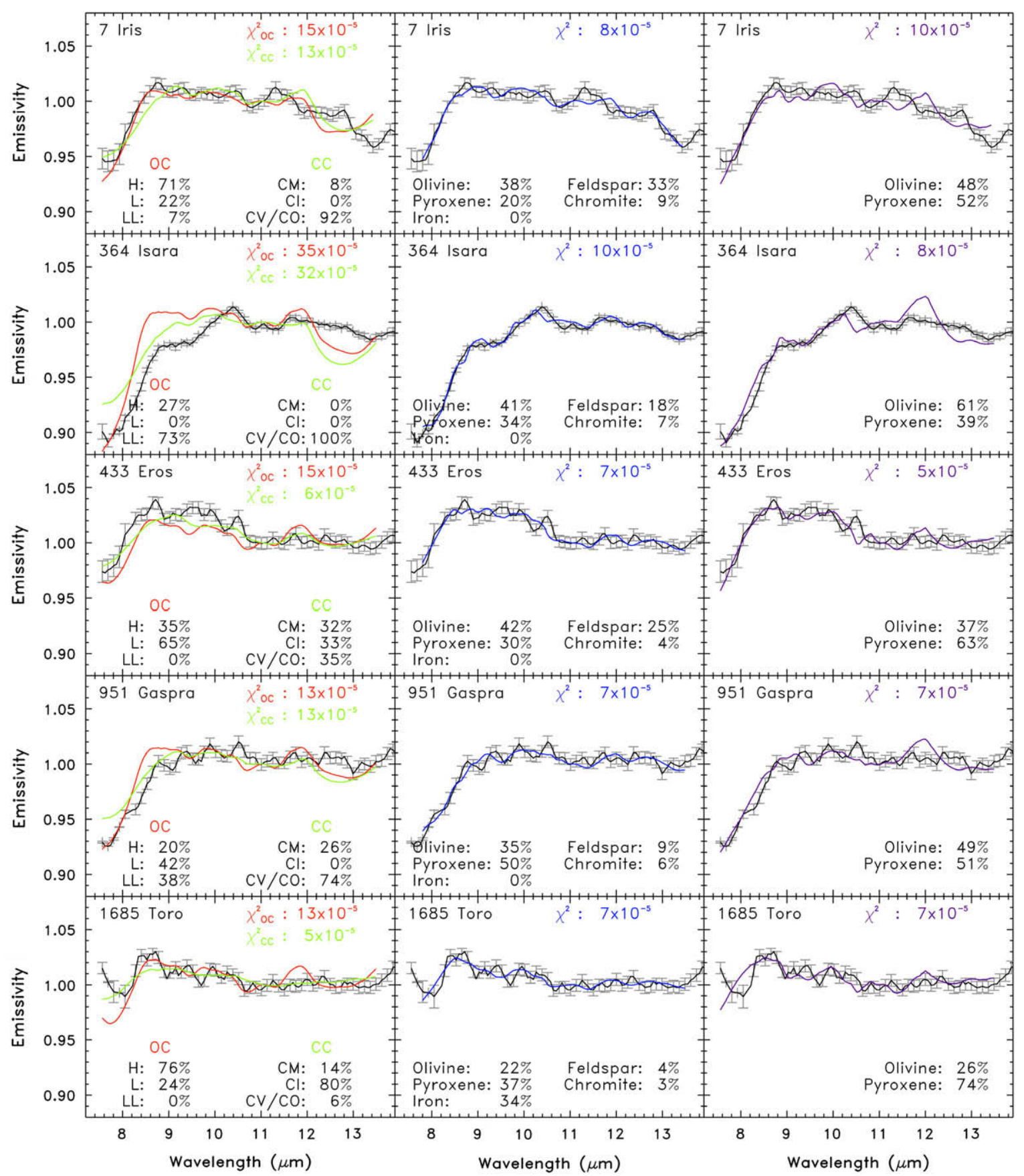

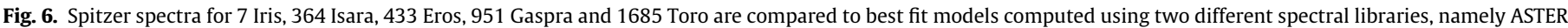

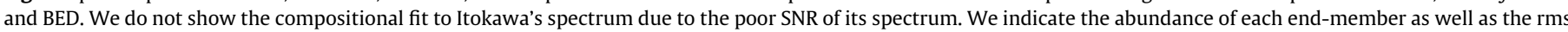

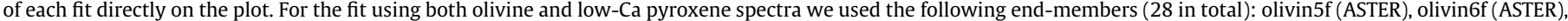

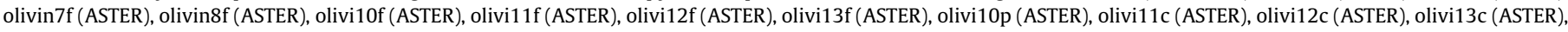

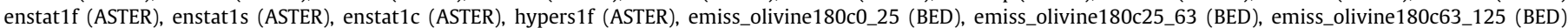

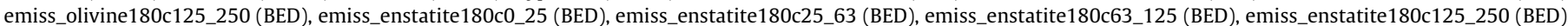

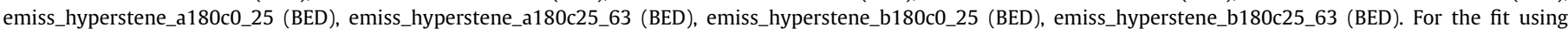

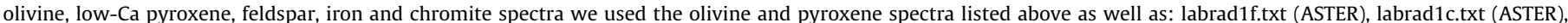

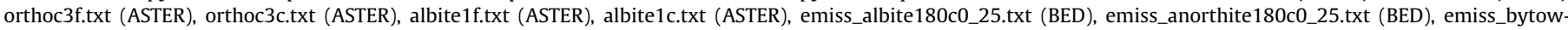

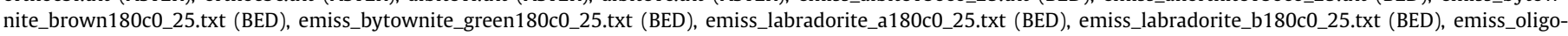
clase180c0_25.txt (BED), emiss_orthoclase180c0_25.txt (BED), lasc06.txt (Relab), lasc63.txt (Relab), chromi1c.txt (ASTER), chromi1f.txt (ASTER).

\section{Discussion}

The variability in compositional interpretation for otherwise similar asteroids may be attributed to the difficulty to constrain the composition of a small grain-sized planetary surface as found previously by laboratory experiments (Hunt and Logan, 1972;
Mustard and Hays, 1997; Ramsey and Christensen, 1998). For example, Mustard and Hays (1997) showed that by slightly varying the grain size of their sample (the minerals were sieved into five particle size separates of $5-\mu \mathrm{m}$ intervals from $<5 \mu \mathrm{m}$ to 20 $25 \mu \mathrm{m})$ the characteristics of the recorded emissivity changes. The observed changes were the following. Christiansen features 


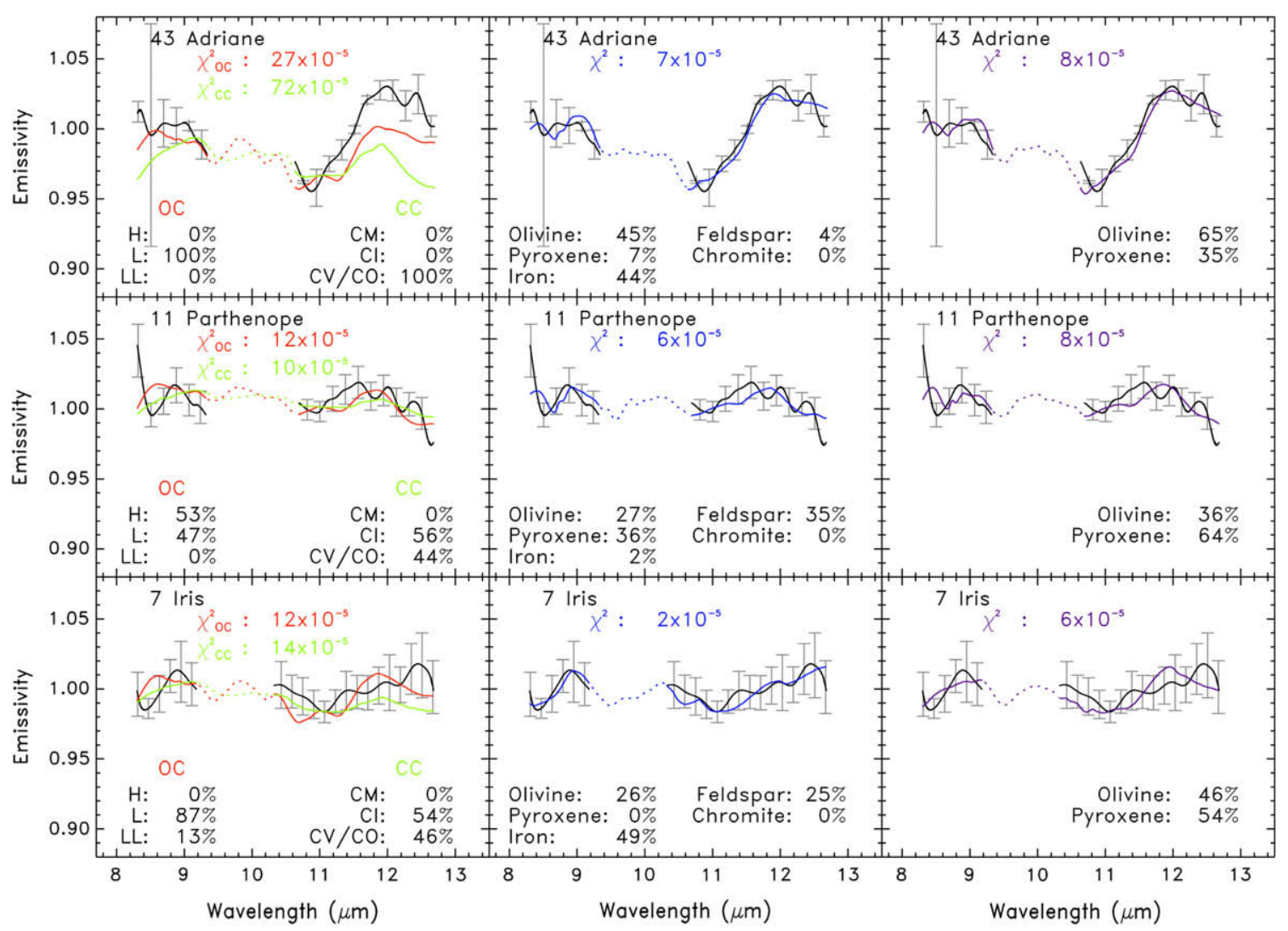

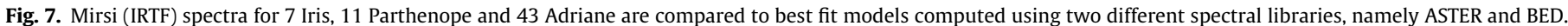
We indicate the abundance of each end-member as well as the rms of each fit directly on the plot.

became saturated, while the restrahlen bands showed continuous decrease in spectral contrast and some change in the shape of the bands with decreasing particle size. The transparency features showed several important changes with decreasing particle size: the spectral contrast increased than decreased, the position of the maximum reflectance of the transparency features shifted systematically to shorter wavelengths, and the symmetry of the features changed.

These important spectral changes observed for small variations of the grain size may allow an explanation for the spectral differences observed among our S-type sample. While we do expect the observed objects to have their surfaces covered by small particles $(<50 \mu \mathrm{m}$, see Dollfus et al., 1977), there is no reason that they should have exactly the same distribution of particle sizes on their surfaces.

Most significantly, these important spectral changes for small variations of the grain size may impede the ability to retrieve the composition in the mid-IR range. The spectral libraries such ASTER and BED provide spectra for grain size intervals that are quite large (0-30 $\mu \mathrm{m}, 0-60 \mu \mathrm{m}, 0-75 \mu \mathrm{m}$ and $75-250 \mu \mathrm{m}$ for ASTER and 0-25 $\mu \mathrm{m}, 25-63 \mu \mathrm{m}, 63-125 \mu \mathrm{m}$ and $125-250 \mu \mathrm{m}$ for BED). Therefore, it is very likely that the grain size of the laboratory samples will not match the average grain size present on the asteroid surfaces which could explain the wrong inferred abundances for olivine and pyroxene. For the interpretation of the actual S-type mid-IR spectra, a preparation of well chosen minerals such olivine and pyroxene into several particle size separates of 5 - $\mu \mathrm{m}$ intervals from $<5 \mu \mathrm{m}$ to $50 \mu \mathrm{m}$ could certainly help improving the fitting results and could help us to appreciate more accurately the usefulness of the mid-IR range for constraining the composition of asteroids.

Another point is that various minerals are spectrally active in different wavelength ranges. One possibility is that the $\mathrm{ol} /(\mathrm{ol}+\mathrm{px})$ ratio could be different between the VNIR and MIR because each wavelength range is sensing different surface components (or sensing them with different strengths). It is precisely to test this possibility that we performed the fitting of case c (including all the minerals that are present in ordinary chondrite meteorites). While we find that there is some variation in the $\mathrm{ol} /(\mathrm{ol}+\mathrm{px})$ value derived from the mid-IR spectra between cases $b$ and $c$, the variation is not enough to explain the discrepancy between the VNIR and MIR analyses.

Finally, we would like to recall one clear limitation of the technique employed here, namely the fact that we did not consider the spectral effects of space weathering processes when fitting the mid-IR asteroid spectra (see Chapman (2004) for a review on these processes). While their spectral effects are pretty well characterized for the VNIR range (true for OC-like material), little has been published yet concerning their effects over the mid-IR range. Brucato et al. (2004) reported the results of ion irradiation experiments on forsterite; they collected mid-IR spectra before and after irradiation. They found that the effect in the mid-IR is to begin to "amorphousize" the surface. This makes sense, since space weathering is thought to create glassy coating (with the embedded nanophase iron). So, to first order, the effect would be to simply reduce the spectral contrast of the restrahlen bands. Besides this paper, no other work related to this topic has been published yet. We therefore encourage researchers performing space weathering experiments to not only limit their spectral measurements (before and after irradiation) to the VNIR range but to extend their measurements to the mid-IR range.

\section{Conclusion}

In this work, we examined a set of eight compositionally similar S-type asteroids to explore their similarities and diversities in the 
mid-IR. We report an unexpected variability among their midinfrared spectra. Compositional fitting making use of only their mid-IR spectra yields surprising alternative conclusions: (1) these objects are not "compositionally similar" as the inferred abundances of their main surface minerals (olivine and pyroxene) differ from one another by $\sim 35 \%$ and (2) carbonaceous chondrite and ordinary chondrite meteorites provide an equally good match to each asteroid spectrum.

Rather than favor either of these two surprising alternative conclusions, we consider it much more likely that our observations are a confirmation of the laboratory work of Ramsey and Christensen (1998), now measurable on asteroids. Based on the Ramsey and Christensen (1998) results, we interpret the mid-IR measurement variability (of an otherwise demonstrated "compositionally consistent" sample of eight S-type asteroids) to be physically caused by differences in surface particle size. For asteroids, it thus appears that mid-infrared measurements are a powerful tool for inter-comparison of surface properties for objects of known compositions (i.e. compositions constrained by other measurements, such as VNIR spectra and/or albedos). Compositionally constrained objects yielding compatible mid-IR measurements (and hence consistent mid-IR compositional interpretations) likely have large particle sizes on their surfaces. Divergent compositional interpretations from mid-IR measurements, where the compositions are otherwise constrained and similar, are more likely indicative of surfaces dominated by particles at or below the $8-13 \mu \mathrm{m}$ scale of mid-IR radiation. Thus for mid-IR measurements of objects whose surface properties are not known, a reliable compositional interpretation based solely on linear deconvolution of mid-IR measurements with existing spectral libraries is problematic. Finally, we stress that space weathering effects may also strongly contribute to the reduction of the spectral contrast. This can be directly tested via new laboratory irradiation experiments.

\section{Acknowledgments}

We thank Lucy F. Lim and an anonymous reviewer for their constructive comments.

\section{References}

Allen, D.A., 1970. Infrared diameter of Vesta. Nature 227, 158-159.

Barucci, M.A., and 12 colleagues, 2002. 10 Hygiea: ISO infrared observations. Astron. Astrophys. 156, 202-210.

Barucci, M.A., and 10 colleagues. 2008. Asteroids 2867 Steins and 21 Lutetia: Surface composition from far infrared observations with the Spitzer Space Telescope. Astron. Astrophys, 477, 665-670.

Binzel, R.P., Rivkin, A.S., Thomas, C.A., Vernazza, P., Burbine, T.H., Demeo, F.E., Bus, S.J., Tokunaga, A.T., Birlan, M., 2009. Spectral properties and composition of potentially hazardous Asteroid (99942) Apophis. Icarus 200, 480-485.

Brucato, J.R., Strazzulla, G., Baratta, G., Colangeli, L., 2004. Forsterite amorphisation by ion irradiation: Monitoring by infrared spectroscopy. Astron. Astrophys. 413, 395-401.

Brunetto, R., Vernazza, P., Marchi, S., Birlan, M., Fulchignoni, M., Orofino, V. Strazzulla, G., 2006. Modeling asteroid surfaces from observations and irradiation experiments: The case of 832 Karin. Icarus 184, 327-337.

Bus, S.J., 1999. Compositional Structure in the Asteroid Belt: Results of a Spectroscopic Survey. Ph.D. Thesis.

Bus, S.J., Binzel, R.P., 2002a. Phase II of the Small Main-belt Asteroid Spectroscopic Survey: The observations. Icarus 158, 106-145.

Bus, S.J., Binzel, R.P., 2002b. Phase II of the Small Main-belt Asteroid Spectroscopic Survey: A feature-based taxonomy. Icarus 158, 146-177.

Bus, S.J., Denault, A.J., Rayner, J.T., Binzel, R.P., Birlan, M., 2002. Remote observing at the NASA Infrared Telescope Facility (IRTF). SPIE 4845, 94-99.

Chapman, C.R., 2004. Space weathering of asteroid surfaces. Annu. Rev. Earth Planet. Sci., 539-567.

Christensen, P.R., Bandfield, J.L., Hamilton, V.E., Howard, D.A., Lane, M.D., Piatek, J.L., Ruff, S.W., Stefanov, W.L., 2000. A thermal emission spectral library of rockforming minerals. J. Geophys. Res. 105, 9735-9740.

Cloutis, E.A., Gaffey, M.J., Jackowski, T.L., Reed, K.L., 1986. Calibrations of phase abundance, composition, and particle size distribution for olivineorthopyroxene mixtures from reflectance spectra. J. Geophys. Res. 91, 641-653.
Cohen, M., Davies, J.K., 1995. Spectral irradiance calibration in the infrared - V. The role of UKIRT and the CGS3 spectrometer. MNRAS 276, 715-722.

Cohen, M., Walker, R.G., Witteborn, F.C., 1992. Spectral irradiance calibration in the infrared. II - Alpha Tau and the recalibration of the IRAS low resolution spectrometer. Astron. J. 104, 2030-2044.

Cohen, M., Walker, R.G., Carter, B., Hammersley, P., Kidger, M., Noguchi, K., 1999 Spectral irradiance calibration in the infrared. X. A self-consistent radiometric all-sky network of absolutely calibrated stellar spectra. Astron. J. 117, 18641889.

Cruikshank, D., Hartmann, W.K., 1984. The meteorite-asteroid connection: Two olivine-rich asteroids. Science 223, 281-283.

Demeo, F.E., Binzel, R.P., Slivan, S., Bus, S.J., 2009. An extension of the Bus asteroid taxonomy into the near-infrared. Icarus 202, 160-180.

Dermott, S.F., Nicholson, P.D., Kim, Y., Wolven, B., Tedesco, E.F., 1988. The impact of IRAS on asteroidal science. Lect. Not. Phys. 297, 3-18.

Deutsch, L.K., Hora, J.L., Adams, J.D., Kassis, M., 2003. MIRSI: A mid-infrared spectrometer and imager. SPIE 4841, 106-116.

Dollfus, A., Geake, J.E., Mandevill, J.C., Zellner, B., 1977. The nature of asteroid surfaces from optical polarimetry. In: Delsemme, A.H. (Ed.), Comets, Asteroids, Meteorites. University of Toledo Press, Toledo, pp. 243-251.

Donaldson Hanna, K.L., Sprague, A.L., 2009. Vesta and the HED meteorites: Midinfrared modeling of minerals and their abundances. Meteorit. Planet. Sci. 44, $1755-1770$.

Donaldson Hanna, K.L., Sprague, A.L., Kozlowski, R.W.H., Boccafolo, K., Warell, J., 2007. Mercury and the Moon: Initial findings from mid-infrared spectroscopic measurements of the surface. Lunar Planet. Sci. Abstract 1338.

Dotto, E., and 10 colleagues, 2000. ISO results on bright main belt asteroids: PHT-S observations. Astron. Astrophys. 358, 1133-1141.

Dotto, E., Barucci, M.A., Müller, T.G., Brucato, J.R., Fulchignoni, M., Mennella, V., Colangeli, L., 2002. ISO observations of low and moderate albedo asteroids. PHT$\mathrm{P}$ and PHT-S results. Astron. Astrophys. 393, 1065-1072.

Emery, J.P., Lim, L.F., McConnochie, T.H., 2006a. Mid-IR Spectroscopy of M asteroids with the Spitzer Space Telescope: How Many are Really Differentiated Cores? L PI Contribution No. 1335, 37-38.

Emery, J.P., Cruikshank, D.P., van Cleve, J., 2006b. Thermal emission spectroscopy $(5.2-38 \mu \mathrm{m})$ of three Trojan asteroids with the Spitzer Space Telescope: Detection of fine-grained silicates. Icarus 182, 496-512.

Emery, J.P., Lim, L.F., Marchis, F., Cruikshank, D.P., 2008. Asteroids and Centaurs: Silicate Emission in the Thermal Infrared. ACM, LPI Contribution No. 1405.

Feely, K.C., Christensen, P.R., 1999. Quantitative compositional analysis using thermal emission spectroscopy: Application to igneous and metamorphic rocks. J. Geophys. Res. 104, 24195-24210.

Feierberg, M.A., Witteborn, F.C., Lebofsky, L.A., 1983. Detection of silicate emission features in the 8- to 13-micron spectra of main belt asteroids. Icarus 56, 393-397.

Gaffey, M.J., Burbine, T.H., Piatek, J.L., Reed, K.L., Chaky, D.A., Bell, J.F., Brown, R.H., 1993. Mineralogical variations within the S-type asteroid class. Icarus 106, 573602.

Gaffey, M.J., Cloutis, E.A., Kelley, M.S., Reed, K.L., 2002. Mineralogy of asteroids. In: Bottke, E.F., Jr., Cellino, A., Paolicchi, P., Binzel, R.P. (Eds.), Asteroids III. University of Arizona Press, Tucson, pp. 183-204.

Gillespie, A.R., 1992. Spectral mixture analysis of multispectral thermal infrared images. Remote Sens. Environ. 42, 137-145.

Gillett, F.C., Merrill, K.M., 1975. 7.5-13.5 micron spectra of Ceres and Vesta. Icarus $26,358-360$.

Green, S.F., Eaton, N., Aitken, D.K., Roche, P.F., Meadows, A.J., 1985. 8- to 13-micron spectra of asteroids. Icarus $62,282-288$.

Hamilton, V.E., Christensen, P.R., 2000. Determining the modal mineralogy of mafic and ultramafic igneous rocks using thermal emission spectroscopy. J. Geophys. Res. 105, 9717-9734.

Hansen, O.L., 1976. Thermal emission spectra of 24 asteroids and the Galilean satellites. Icarus 27, 463-471.

Hapke, B., 1993. Combined theory of reflectance and emittance spectroscopy. In: Pieters, C.M., Englert, P.A.J. (Eds.), Remote Geochemical Analysis: Elemental and Mineralogical Composition. Cambridge University Press, New York, pp. 31-42.

Helbert, J., Moroz, L.V., Maturilli, A., Bischoff, A., Warell, J., Sprague, A., Palomba, E. 2007. A set of laboratory analogue materials for the MERTIS instrument on the ESA BepiColombo mission to Mercury. Adv. Space Res. 40, 272-279.

Houck, J.R., and 34 colleagues, 2004. The InfraRed Spectrograph (IRS) on the Spitzer Space Telescope. Astrophys. J. 154, 18-24.

Hunt, G.R., Logan, L.M., 1972. Variation of single particle mid-infrared emission spectrum with particle size. Appl. Opt. 11, 142-147.

Hunt, G.R., Vincent, R.K., 1968. The behavior of spectral features in the infrared emission from particulate surfaces of various grain sizes. J. Geophys. Res. 73, 6039-6046.

Hutchison, R., 2004. Meteorites: A Petrologic, Chemical and Isotopic Synthesis. Cambridge University Press, Cambridge, UK

Kozlowski, R.W., Donaldson Hanna, K., Sprague, A.L., Grosse, F.A., Boop, T.S., Warell, J., Boccafola, K., 2007. The Moon: Determining Minerals and their Abundances with Mid-IR Spectral Deconvolution II. DPS Abstract, p. 431.

Lagerros, J.S.V., 1998. Thermal physics of asteroids. IV. Thermal infrared beaming. Astron. Astrophys. 332, 1123-1132.

Lebofsky, L.A., Sykes, M.V., Tedesco, E.F., Veeder, G.J., Matson, D.L., Brown, R.H. Gradie, J.C., Feierberg, M.A., Rudy, R.J., 1986. A refined 'standard' thermal model for asteroids based on observations of 1 Ceres and 2 Pallas. Icarus 68, 239-251.

Lim, L.F., Emery, J.P., 2006. A Spitzer IRS Survey of Hydrated and Non-Hydrated M asteroids: Preliminary 5-13 micron Results. DPS Abstract, p. 626. 
Lim, L.F., McConnochie, T.H., Bell, J.F., Hayward, T.L., 2005a. Thermal infrared (8$13 \mu \mathrm{m}$ ) spectra of 29 asteroids: The Cornell Mid-Infrared Asteroid Spectroscopy (MIDAS) Survey. Icarus 173, 385-408

Lim, L.F., Emery, J.P., McConnochie, T.H., 2005b. Thermal Infrared (8-13 micron) Spectra of the NEA 2100 Ra-Shalom. DPS Abstract, p. 629.

Lim, L.F., Emery, J.P., McConnochie, T.H., 2006. Mid-IR spectroscopy of $M$ asteroids with the spitzer IRS: Preliminary results. Lunar Planet. Sci. Abstract 2148.

Lim, L.F., Emery, J.P., Moskovitz, N.A., Stewart, H., Marchis, F., 2008. Spitzer IRS Spectra of Basaltic Asteroids: Preliminary Results. DPS Abstract, p. 436.

Lim, L.F., Emery, J.P., Moskovitz, N.A., 2009. Diogenite-like features in the Spitzer IRS (5-35 $\mu \mathrm{m})$ spectrum of 956 Elisa. Lunar Planet. Sci. Abstract 2204.

Lord, S.D., 1992. A new software tool for computing Earth's atmospheric transmission of near- and far-infrared radiation. NASA Technical Memorandum 103957.

Lyon, R.J.P., 1964. Evaluation of Infrared Spectrophotometry for Compositional Analysis of Lunar and Planetary Soils. Part II: Rough and Powdered Surfaces. NASA-CR-100, Washington, DC.

Marchis, F., Hardersen, P.S., Emery, J.P., Descamps, P., Reddy, V., Lim, L.F., 2008. Composition of the binary main-belt Asteroid (22) Kalliope. Lunar Planet. Sci. Abstract 1391

Matson, D.L., Veeder, G.J., Tedesco, E.F., Lebofsky, L.A., Walker, R.G., 1986. IRAS survey of asteroids. Adv. Space. Res. 6, 47-56.

Maturilli, A., Helbert, J., Witzke, A., Moroz, L., 2006. Emissivity measurements of analogue materials for the interpretation of data from PFS on Mars Express and MERTIS on Bepi-Colombo. Planet. Space Sci. 54, 1057-1064.

Maturilli, A., Helbert, J., Moroz, L., 2008. The Berlin emissivity database (BED). Planet. Space Sci. 56, 420-425.

McCord, T.B., Adams, J.B., Johnson, T.V., 1970. Asteroid Vesta: Spectral reflectivity and compositional implications. Science 168, 1445-1447.

Milam, K.A., McSween, H.Y., Hamilton, V.E., Moersch, J.M., Christensen, P.R., 2004 Accuracy of plagioclase compositions from laboratory and Mars spacecraft thermal emission spectra. J. Geophys. Res. 109, E04001.

Milam, K.A., McSween, H.Y., Christensen, P.R., 2007. Plagioclase compositions derived from thermal emission spectra of compositionally complex mixtures: Implications for martian feldspar mineralogy. J. Geophys. Res. 112, E10005.

Moersch, J.E., Christensen, P.R., 1995. Thermal emission from particulate surfaces: A comparison of scattering models with measured spectra. J. Geophys. Res. 100, 7465-7477.

Morrison, D., 1973. Determination of radii of satellites and asteroids from radiometry and photometry. Icarus $19,1-14$.

Morrison, D., 1974. Radiometric diameters and albedos of 40 Asteroids. Astrophys. J. $194,203-212$

Morrison, D., 1977. Asteroid sizes and albedos. Icarus 31, 185-220.

Mueller, T.G., Lagerros, J.S.V., 1998. Asteroids as far-infrared photometric standards for ISOPHOT. Astron. Astrophys. 338, 340-352.

Mustard, J.F., Hays, J.E., 1997. Effects of hyperfine particles on reflectance spectra from 0.3 to $25 \mu \mathrm{m}$. Icarus $125,145-163$.
Nesvorný, D., Vokrouhlický, D., Morbidelli, A., Bottke, W.F., 2009. Asteroidal source of L chondrite meteorites. Icarus 200, 698-701.

Ramsey, M.S., Christensen, P.R., 1998. Mineral abundance determination: Quantitative deconvolution of thermal emission spectra. J. Geophys. Res. 103, 577-596.

Ramsey, M.S., Fink, J.H., 1999. Estimating silicic lava vesicularity with thermal remote sensing: A new technique for volcanic mapping and monitoring. Bull. Volcanol. 61, 32-39.

Ramsey, M.S., Christensen, P.R., Lancaster, N., Howard, D.A., 1999. Identification of sand sources and transport pathways at the Kelso dunes, California, using thermal infrared remote sensing. Geol. Soc. Am. Bull. 111, 646-662.

Rayner, J.T., Toomey, D.W., Onaka, P.M., Denault, A.J., Stahlberger, W.E., Vacca, W.D., Cushing, M.C., Wang, S., 2003. A medium-resolution 0.8-5.5 micron spectrograph and imager for the NASA Infrared Telescope Facility. PASP 115, 362-382.

Rivkin, A.S., Howell, E.S., Vilas, F., Lebofsky, L.A., 2002. Hydrated minerals on asteroids: The astronomical record. In: Bottke, W.F., Jr., Cellino, A., Paolicchi, P., Binzel, R.P. (Eds.), Asteroids III. University of Arizona Press, Tucson, pp. 235253

Rivkin, A.S., Binzel, R.P., Sunshine, J., Bus, S.J., Burbine, T.H., Saxena, A., 2004. Infrared spectroscopic observations of 69230 Hermes (1937 UB) and (19356) 1997GH3: Possible unweathered endmembers among ordinary chondrite analogs. Icarus $172,408-414$

Rivkin, A.S., Volquardsen, E.L., Clark, B.E., 2006. The surface composition of Ceres: Discovery of carbonates and iron-rich clays. Icarus 185, 563-567.

Salisbury, J.W., D’Aria, D.M., Jarosewich, E., 1991. Midinfrared (2.5-13.5 microns) reflectance spectra of powdered stony meteorites. Icarus 92, 280-297.

Shkuratov, Y., Starukhina, L., Hoffmann, H., Arnold, G., 1999. A model of spectral albedo of particulate surfaces: Implications for optical properties of the Moon. Icarus 137, 235-246.

Sprague, A.L., Donaldson Hanna, K.L., Kozlowski, R.W.H., Helbert, J., Maturilli, A., Warell, J.B., Hora, J.L., 2009. Spectral emissivity measurements of Mercury's surface indicate Mg- and Ca-rich mineralogy, K-spar, Na-rich plagioclase, rutile, with possible perovskite, and garnet. Planet. Space Sci. 57, 364-383.

Sunshine, J.M., Bus, S.J., Corrigan, C.M., McCoy, T.J., Burbine, T.H., 2007. Olivinedominated asteroids and meteorites: Distinguishing nebular and igneous histories. Meteorit. Planet. Sci. 42, 155-170.

Tedesco, E.F., Matson, D.L., Veeder, G.J., Lebofsky, L.A., 1988. IRAS observations of asteroids. Lect. Not. Phys. 297, 19-26.

Tedesco, E.F., Noah, P.V., Noah, M., Price, S.D., 2002. The supplemental IRAS Minor Planet Survey. Astron. J. 123, 1056-1085.

Thomson, J.L., Salisbury, J.W., 1993. The mid-infrared reflectance of mineral mixtures (7-14 $\mu \mathrm{m})$. Remote Sens. Environ. 45, 1-13.

Vernazza, P., Binzel, R.P., Thomas, C.A., Demeo, F.E., Bus, S.J., Rivkin, A.S., Tokunaga, A.T., 2008. Compositional differences between meteorites and near-Earth asteroids. Nature $454,858-860$.

Vernazza, P., Brunetto, R., Binzel, R.P., Perron, C., Fulvio, D., Strazzulla, G., Fulchignoni, M., 2009. Plausible parent bodies for enstatite chondrites and mesosiderites: Implications for Lutetia's fly-by. Icarus 202, 477-486. 